\title{
Saúde do trabalhador e morbimortalidade por covid-19 entre os profissionais da saúde no Brasil
}

\begin{abstract}
RESUMO | OBJETIVO: Avaliar a interface saúde do trabalhador e a morbimortalidade por COVID-19 entre os Trabalhadores da Saúde no Brasil. MÉTODO: estudo epidemiológico descritivo com abordagem quantitativa realizado em julho de 2021. Utilizouse dados on-line provenientes do Painel Interativo Impacto COVID-19 da Plataforma da Rede Nacional de Atenção Integral à Saúde do Trabalhador. Por meio do Painel Interativo Impacto Covid-19 evidenciou-se que é alto o risco de contágio de infecções e doenças entre os trabalhadores da saúde. RESULTADOS: Considera-se maior o risco quanto maior seja a proximidade física para realização de suas atividades laborais em um nível alto de contágio entre 70\% a 100\%. CONCLUSÂO: Os achados reforçam o quanto é importante a Saúde do Trabalhador e deve ser observada para fomentar discussão acerca dos fatores que implicam na saúde do trabalho dos profissionais da saúde e estratégias para dar o suporte necessário para que possam exercer seu trabalho com minimização dos riscos.
\end{abstract}

Descritores: Saúde do Trabalhador; Morbimortalidade; Covid-19.

\begin{abstract}
OBJECTIVE: To assess the worker's health interface and COVID-19 morbidity and mortality among health workers in Brazil. METHOD: descriptive epidemiological study with a quantitative approach carried out in July 2021. Online data from the COVID-19 Impact Interactive Panel of the Platform of the National Network for Integral Attention to Workers' Health were used. Through the Covid-19 Impact Interactive Panel, it became clear that the risk of contagion of infections and diseases among health workers is high. RESULTS: The greater the risk the greater the physical proximity to carry out their work activities at a high level of contagion between $70 \%$ and $100 \%$. CONCLUSION: The findings reinforce how important Worker's Health is and should be observed to encourage discussion about the factors that affect the health at work of health professionals and strategies to provide the necessary support so that they can perform their work with minimization of risks.
\end{abstract}

Keywords: Occupational Health; Morbidity and Mortality; Covid-19.

RESUMEN | OBJETIVO: Evaluar la interfaz de salud del trabajador y la morbilidad y mortalidad por COVID-19 entre los trabajadores de la salud en Brasil. MÉTODO: estudio epidemiológico descriptivo con enfoque cuantitativo realizado en julio de 2021. Se utilizaron datos en línea del Panel Interactivo de Impacto COVID-19 de la Plataforma de la Red Nacional para la Atención Integral a la Salud de los Trabajadores. A través del Panel Interactivo Impacto Covid-19, quedó claro que el riesgo de contagio de infecciones y enfermedades entre los trabajadores de la salud es alto. RESULTADOS: A mayor riesgo mayor proximidad física para realizar sus actividades laborales con un alto nivel de contagio entre $70 \%$ y $100 \%$. CONCLUSIÓN: Los hallazgos refuerzan cuán importante es y debe ser observada la Salud del Trabajador para incentivar la discusión sobre los factores que afectan la salud en el trabajo de los profesionales de la salud y las estrategias para brindar el apoyo necesario para que puedan desempeñar su trabajo con minimización de riesgos.

Palabras claves: Salud Laboral; Morbimortalidad; Covid-19.

\section{Silvana Maria de Oliveira Sousa}

Enfermeira, especialista em Urgência e Emergência em Enfermagem. Mestranda Programa de Pós-Graduação em Cuidados Clínicos em Enfermagem e Saúde - PPCCLIS, da Universidade Estadual do Ceará. Integrante do Grupo de Pesquisa do CNPq - Políticas, Saberes e Práticas em Enfermagem e Saúde Coletiva. Fortaleza-CE, Brasil. ORCID: 0000-0003-4630-4766

\section{Jéssica Costa Brito Pacheco Moura}

Enfermeira, especialista em Terapia Intensiva. Coordenadora de Enfermagem do Serviço de Emergência pediátrica no Hospital Regional Norte. Mestranda Programa de Pós-Graduação em Cuidados Clínicos em Enfermagem e Saúde, da Universidade Estadual do Ceará. Professora substituta do Curso de Enfermagem da Universidade Vale do Acaraú.

ORCID: 0000-0001-9807-6668

\section{Ana Vitória Pinheiro Neves}

Acadêmica de Enfermagem na Universidade Estadual do Ceará (UECE). Bolsista de Iniciação Científica do Programa Institucional de Bolsas de Iniciação científica (IC/PIBIC). Integrante do Laboratório de Práticas Coletivas em Saúde. Fortaleza-CE, Brasil. ORCID: 0000-0001-7284-8885

\section{Lucilane Maria Sales da Silva}

Enfermeira, Pós-doutorado em Enfermagem pela Escola de Enfermagem Anna Nery/Universidade Federal do Rio de Janeiro. Professora associada da Fundação Universidade Estadual do Ceará. Avaliadora de cursos de graduação e de técnico de Enfermagem pelo Conselho de Educação do Estado do Ceará e pelo INEP/MEC. Vice-líder do Grupo de Pesquisa do CNPq - Políticas, Saberes e Práticas em Enfermagem e Saúde Coletiva.

ORCID: 0000-0002-3850-8753
Recebido em: 11/11/2021

Aprovado em: 25/01/2022

INTRODUÇÃO

A atenção à Saúde do Trabalhador é assunto previsto em lei, embasada na Constituição/88 e na Lei Orgânica da Saúde/90. No âmbito do SUS desde 2009, dispõe da Rede Nacional de Atenção Integral à Saúde do Trabalhador (RENAST). Essa Rede se articula com as Secretarias de Saúde a nível Federal, Estadual e Municipal, além de outros órgãos executores de ações relacionadas com a Saúde do Trabalhador. Dentre algumas funções integra a rede de serviços do SUS, voltados à promoção, à assistên- 
cia e à vigilância, para o desenvolvimento das ações de Saúde do Trabalhador ${ }^{1}$.

Sobre a Vigilância da saúde do Trabalhador atualmente a RENAST de forma integrada com os Centros de Referência em Saúde do Trabalhador (CERESTS) têm desenvolvido ações que favorecem a integralidade de ações de saúde do trabalhador e o controle social para o monitoramento e direcionamento de condutas para os trabalhadores na situação catastrófica da ocorrência da Pandemia COVID-192.

A qualidade de saúde do trabalho depende de vários aspectos, envolve ambiente de trabalho, possibilidades do profissional exercer suas funções. A qualidade da saúde do trabalhador no Brasil, já fragilizada pelas perdas relevantes de direitos trabalhistas e previdenciários, somadas às desigualdades sociais preexistentes se tornou ainda mais crítica e ameaçada perante o caos advindo com a pandemia COVID-193. Assim, é imprescindível que haja atenção integral e monitoramento da situação de saúde desses profissionais.

Com o advindo da doença COVID-19 que surgiu em Muhan-China em dezembro de 2019 os governos dos países precisaram de forma repentina traçar estratégias para lidar com a explosão de casos da COVID-19 por ser uma doença de alta transmissibilidade e com altos números de óbitos em todo o mundo. Nesse contexto mundial de milhões de óbitos houve muitas pessoas em idade economicamente ativa que morreram. Enquanto outro montante de trabalhadores teve que dar continuidade às suas atividades laborais enfrentando o vírus em seus ambientes e jornadas de trabalho2.

Em um contexto pandêmico, deflagrada a velocidade de disseminação de casos, os sistemas de informação desempenham um papel essencial no compilado dos dados com a rapidez exigida pela situação. É relevante analisar os Sistemas de Saúde para o acompanhamento da epidemiologia. O Bra-
A qualidade de saúde do trabalho depende de vários aspectos, envolve ambiente de trabalho, possibilidades do profissional exercer

suas funções. A qualidade da saúde do trabalhador no Brasil, já fragilizada pelas perdas relevantes de direitos trabalhistas e previdenciários, somadas às desigualdades sociais preexistentes se tornou ainda mais crítica e ameaçada perante o caos advindo com a pandemia COVID-19 sil conta com sistemas de informação que, referente à pandemia COVID-19, desde o seu início teve papel importante direcionando as ações visando garantir a atenção à saúde integral do trabalhador.

Assim, para melhor entendimento de como a epidemiologia da pandemia se comportou entre os profissionais de saúde considerados linha de frente ao Combate à COVID-19, objetivou-se avaliar a interface saúde do trabalhador e a morbimortalidade por COVID-19 entre os Profissionais da Saúde no Brasil.

\section{MÉTODO}

Estudo epidemiológico descritivo com abordagem quantitativa. Os estudos descritivos objetivam "determinar a distribuição de doenças ou condições relacionadas à saúde, segundo o tempo, o lugar e/ou as características dos indivíduos" de forma que seja possível investigar "quando, onde e quem adoece" utilizando dados secundários em importantes bancos de dados de abrangência nacional e estadual冈.

Realizou-se o presente estudo em duas etapas: Na primeira ocorreu a exploração do site da Plataforma RENAST. $\mathrm{Na}$ segunda se deu a compilação dos achados e organização dos dados em figuras e tabelas para posterior discussão dos resultados com a literatura pertinente.

$\mathrm{Na}$ Plataforma RENAST buscou-se as produções e registros dos dados epidemiológicos referentes aos profissionais da saúde. Adotou-se como critério de inclusão fontes sobre morbimortalidade correlacionando-os à COVID-19. Excluiu-se dados e registros de outras populações, referentes à outras doenças ou Síndromes Respiratórias Agudas e os boletins anteriores à pandemia. Apesar de não haver na própria plataforma um painel ou boletim de dados atuais dos trabalhadores brasileiros, ela compilou vários links, sites e hotsite com os mais 
diversos temas relacionados à saúde do trabalhador com os dados que eram captados pelas organizações, instituições e pesquisas relacionadas.

Assim na seção "Risco e agravos à saúde do trabalhador, COVID -19" da Plataforma RENAST escolheu-se um painel criado por pesquisadores do Rio de Janeiro. O Painel Interativo Impacto Covid-19 foi criado para avaliação do Risco de Contágio por Ocupação no Brasil. Utilizam como fonte: o "The Workers Who Face the Greatest Coronavirus Risk", a lista de atividades econômicas proveniente da Classificação Nacional de Atividades Econômicas (CNAE) elaborada pelo IBGE, a Relação Anual de Informações Sociais (RAIS) e a Classificação Brasileira de Ocupações (CBO).Os dados da RAIS e da CBO se referem ao registro de todos os profissionais cadastrados na atualização mais recente que datam de 2018 . outros dados, consta os dados de Risco de Contágio por Ocupação, Risco de Contágio por Atividade e Risco de Contágio por Ocupação no Brasil em formato de gráfico de bolhas com a possibilidade de escolha de visualização por categoria profissional e regulagem do nível do risco de contágio凹. No presente estudo os dados de interesse foram extraídos e transportados para o software Microsoft Power Point 2016®, posteriormente organizados e convertidos em imagens.

O presente estudo não dispõe de número de comitê de ética por ter sido realizado com dados secundários e agregados, mas segue conformidade com as diretrizes da ética em pesquisa.

\section{RESULTADOS}

Por meio do Painel Interativo Impacto Covid-19 para avaliação do Risco possível verificar que os profissionais de saúde são uns dos que mais necessitam de proximidade física para realização de suas atividades laborais, que são mais expostos à doenças e infecções e, devido a isso, a maioria estão em um nível entre $70 \%$ a $100 \%$ o que implica em alto nível do risco de contágio demonstrado na Figura 1.

Lima, Costa e Souza (2021) 》 explicam que a avaliação da frequência da exposição é medida em uma escala graduada de zero a cem. O "Nível do risco de contágio" que foi calculado para cada ocupação através de uma média dos valores de três variáveis: 1 - Com que frequência o exercício da ocupação demanda exposição às doenças ou infecções. 2 - Até que ponto o exercício da ocupação exige que o trabalhador realize tarefas em estreita proximidade física com outras pessoas e 3 - O quanto o exercício da ocupa-

\section{Figura 1. Risco de contágio dos profissionais considerando a exposição à doença ou infecções e a proximidade física. Brasil.}

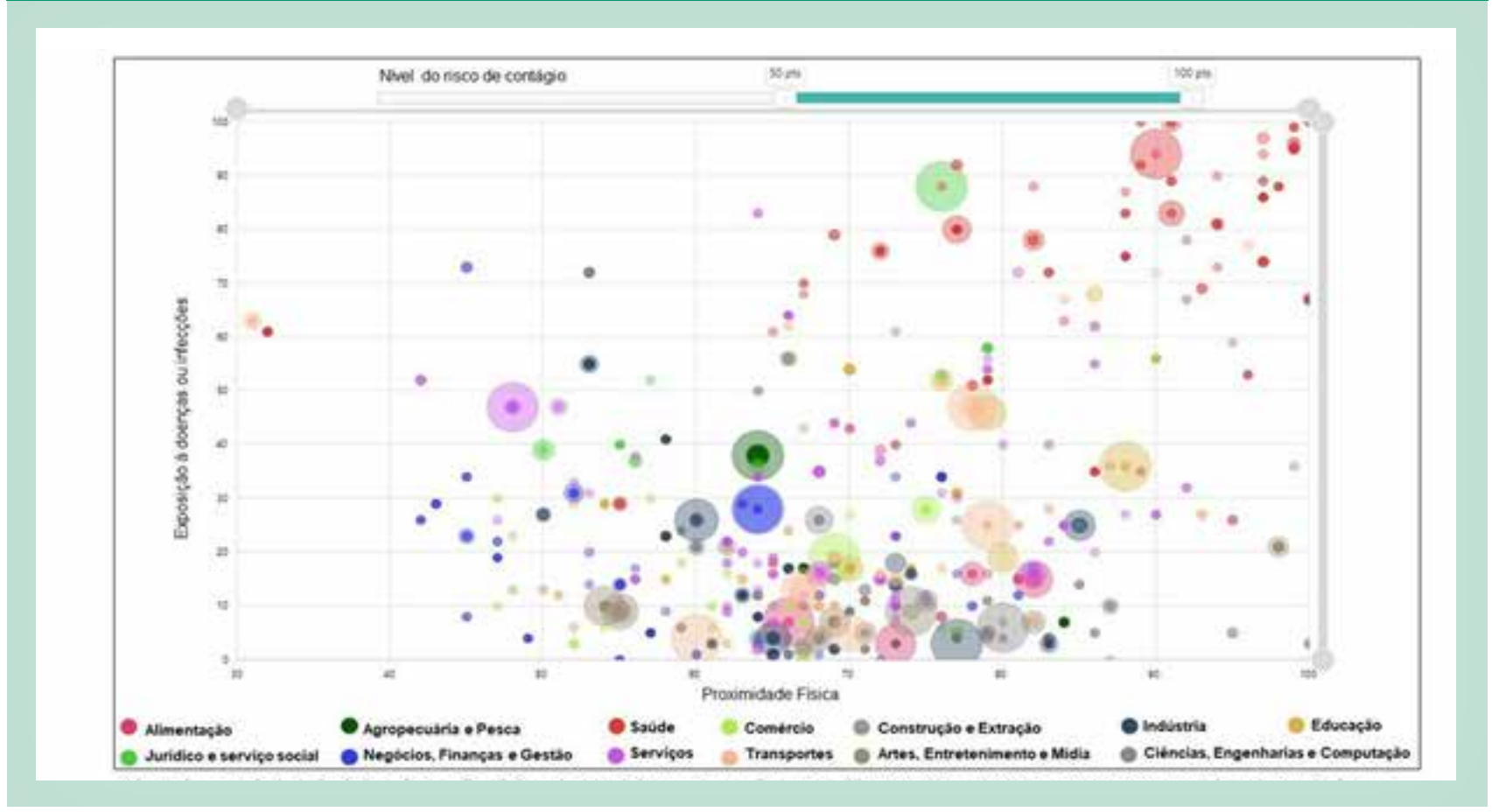

Adaptado a partir do Painel de Informações "Risco de Contágio por Ocupação no Brasil": LIMA, Y. O., COSTA, D. M., SOUZA, J. M. Risco de Contágio por Ocupação no Brasil. Impacto COVID-19, Rio de Janeiro, 26 de Mar. de 2020. Disponível em: https://impactocovid.com.br. Acesso em: 10 de Julho de 2021 
contato com outras pessoas.

No eixo vertical $(Y)$, o gráfico mostra o quanto os trabalhadores de cada uma das ocupações exibidas tendem a se expor às doenças ou infecções nas suas atividades. Nesse eixo vertical, temos ocupações da área da Saúde com valores bem altos de exposição às doenças ou infecções como Enfermeiros, Cirurgiões, Médicos e Técnicos de Enfermagem, sendo todas essas profissões superadas pelo risco de exposição dos profissionais da odontologia que tem os valores de risco mais altos, a exemplo do "Técnico da Saúde Bucal" que contabiliza valor 100, ou seja, exposição diariamente.

Já no seu eixo horizontal (X), o gráfico traz a informação do quanto a execução das atividades das ocupações exige que os trabalhadores fiquem em contato próximo com outras pessoas. Nesse caso, conforme a figura abai- xo, a escala vai de "distante de outras pessoas (mais de 30m)" (por exemplo no caso do médico urologista que tem valor 32 de proximidade) até "muito próximo" (como o fazem os "Cirurgiões-dentistas" com valor 99).

$\mathrm{Na}$ figura 2 pode ser verificado o Índice de Risco de Contágio por Ocupação de todas as áreas de atuação dos trabalhadores em risco de se contaminarem durante suas atividades de trabalho. Percebe-se que o percentual de trabalhadores em risco está concentrado entre os valores de 45 a 65 dos índices de risco de contágio, enquanto os trabalhadores da saúde humana e serviço social estão entre os valores de 70 a 80 e com maior percentual de trabalhadores em risco de contágio ao desenvolver suas ocupações.

O valor apresentado no eixo vertical é calculado pela fórmula de Índice de risco de contágio. Já no eixo hori- zontal, o valor apresentado é a quantidade de trabalhadores sob risco nas atividades econômicas (em percentual). Esse risco pode variar de 0 a 100 , com 0 representando nenhum risco de contágio e 100 representando um altíssimo risco de contágio. São considerados trabalhadores sob risco aqueles cujo risco de contágio tem valor igual ou superior à 60 pontos.

A Figura 3 representa a seleção unicamente do Índice de Risco de Contágio por Ocupação de Trabalhadores da Saúde Humana em risco de se contaminarem durante suas atividades de trabalho. Serve de embasamento para compreensão da dimensão do risco que mesmo estão sujeitos em comparação aos demais.

Os achados revelam que as categorias profissionais mais atuantes nos estabelecimentos de saúde foram as que tiveram maior número de casos confir-

\section{Figura 2. Nível de risco de contágio considerando o Índice de risco de contágio pelo percentualdos trabalhadores em risco, Brasil}

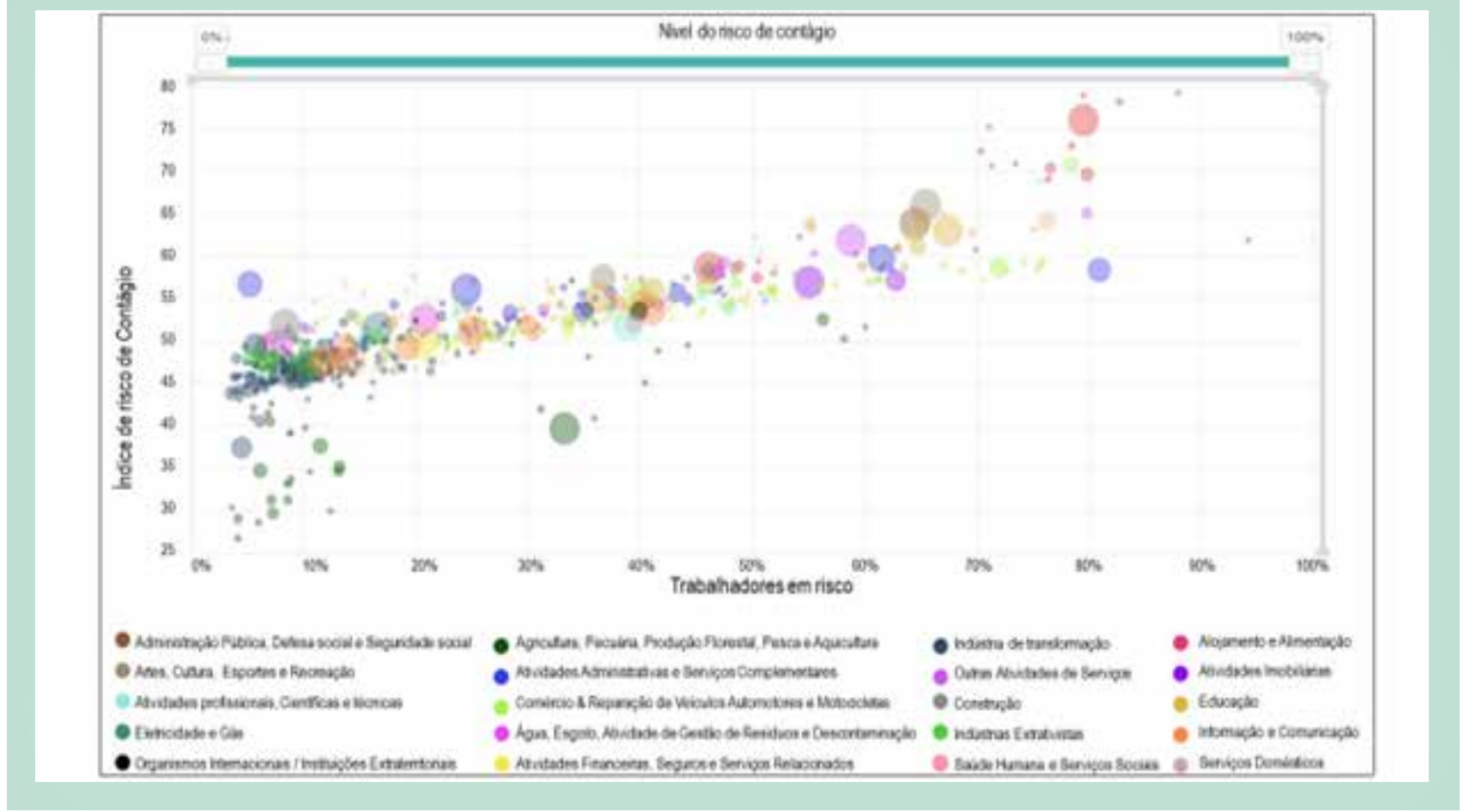

Adaptado a partir do Painel de Informações "Risco de Contágio por Ocupação no Brasil": LIMA, Y. O., COSTA, D. M., SOUZA, J. M. Risco de Contágio por Ocupação no Brasil. Impacto COVID-19, Rio de Janeiro, 26 de Mar. de 2020. Disponível em: https://impactocovid.com.br. Acesso em: 10 de Julho de 2021 
Figura 3. Nível de risco de contágio considerando o Índice de risco de contágio pelo percentual dos trabalhadores da Saúde Humana em risco, Brasil.

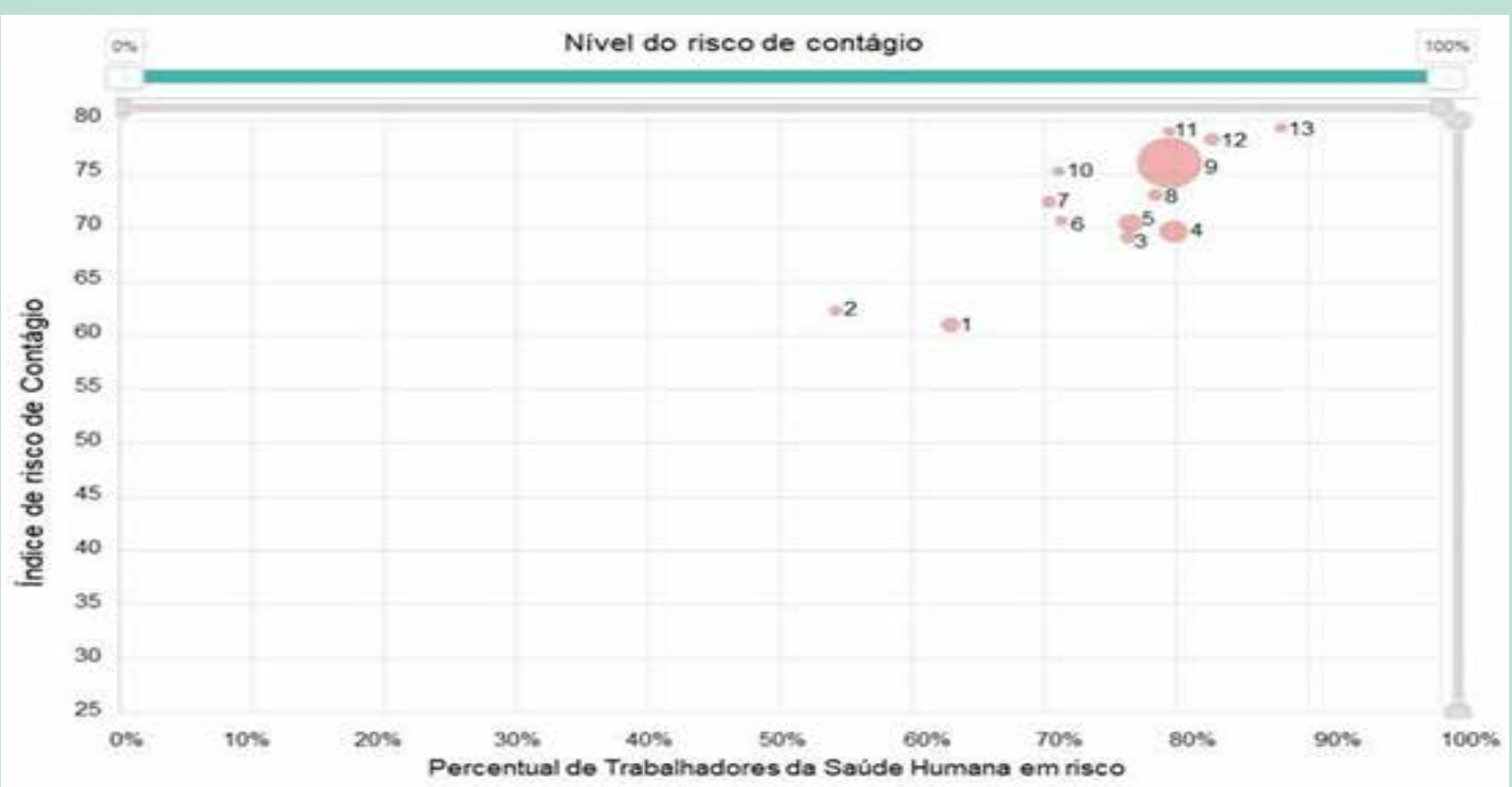

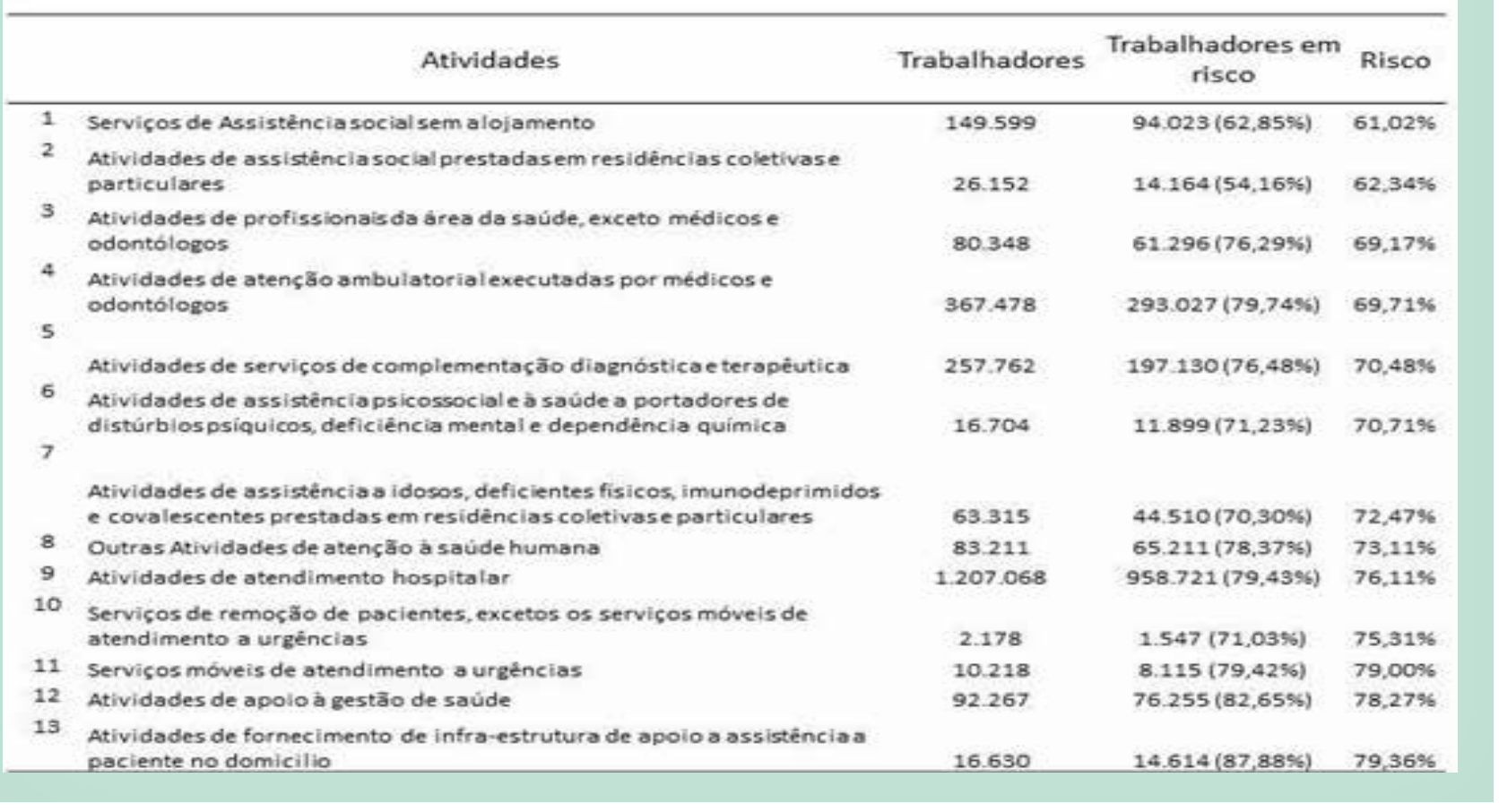

Adaptado a partir do Painel de Informações "Risco de Contágio por Ocupação no Brasil": LIMA, Y. O., COSTA, D. M., SOUZA, J. M. Risco de Contágio por Ocupação no Brasil. Impacto COVID-19, Rio de Janeiro, 26 de Mar. de 2020. Disponível em: https://impactocovid.com.br. Acesso em: 10 de Julho de 2021

mados, o que pode ser relacionado à questão da proximidade e aumento do risco de contágio.

\section{DISCUSSÃO}

Os achados do presente estudo apontam que os profissionais de saúde por realizarem suas funções de assistência com necessidade de proximidade do seu público alvo possuem maior risco de se contaminarem com o SAR- 
S-Cov-2.

Para uma determinada infecção acontecer depende da tríade epidemiológica das características biológicas do patógeno, do ambiente e das características da população. Os profissionais de saúde têm esse risco aumentado devido à maior probabilidade de transmissão em ambientes onde os riscos de infecção são combinados e várias redes de transmissão se cruzam. Autores afirmam que alguns fatores facilitam um maior risco de infecção por causa dos riscos compostos, muitas vezes impulsionados pela dinâmica da rede (contatos frequentes, proximidade e contato prolongado) e determinantes de nível estrutural (como pobreza, ocupação e tamanho do ambiente em que as pessoas precisam estar6.

No início da pandemia com a disseminação da COVID-19, os serviços de saúde ficaram sobrecarregados e os profissionais passaram a enfrentar diariamente estressores ainda maiores, além do aumento intensificado dos riscos à sua própria saúde. À medida em que os hospitais atingiam sua capacidade máxima, os suprimentos, os EPI essenciais para a proteção e indispensáveis para evitar o contágio, se tornaram mais escassos. Esses e muitos outros desafios enfrentados favoreciam o aumento dos riscos potenciais biológicos $\bigotimes$.

Em um estudo incluindo 30 artigos em uma revisão sistemática e 28 em uma meta-análise objetivando descrever os desfechos clínicos e os fatores de risco para infecção por SARS-CoV-2 em profissionais de saúde. Encontraram entre os estudos avaliados que o aumento geral do risco de infecção foi observado em profissionais de saúde da linha de frente em todos os ambientes de saúde em comparação com a comunidade em geral, com um risco mais alto em profissionais de saúde que trabalham em ambientes de internação e lares de idosos. Mas que o uso corretamente das máscaras faciais mostrou ser protetor, e ter usado uma em todos os momentos

diminuiu o risco de infecção8.

Os autores apontam também que avaliando a variável exposição no ambiente de assistência, isoladamente,

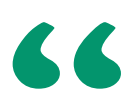

\section{No início da} pandemia com a disseminação da COVID-19, os serviços de saúde ficaram sobrecarregados e os profissionais

passaram a enfrentar diariamente estressores ainda maiores, além do aumento intensificado dos riscos à sua própria saúde

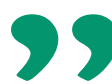

não houve associação entre risco de infecção e tempo de exposição ou distância com pacientes positivos. Consideram que são necessários mais dados para rastrear os riscos contínuos em Profissionais de Saúde à medida que a pandemia evolui e os sistemas de saúde se adaptam e que são necessários mais dados para continuar a compreender a implicação em evolução desta pande- mia na saúde e no bem-estar dos profissionais de saúde a nível internacional区.

Em um estudo transversal com 24.749 profissionais de saúde dos Estados Unidos para investigar quais eram os fatores de riscos associados à soropositividade para o SARS-CoV-2 entre os profissionais de saúde dentro e fora do local de trabalho. Constataram que o contato com um indivíduo com exposição à COVID-19 fora do local de trabalho foi o fator de risco mais forte associado à soropositividade, junto com residir em um endereço situado em um local com maior incidência de COVID-199.

Os autores afirmaram que nenhum dos fatores de trabalho avaliados foi associado à soropositividade. Isso implica que as exposições fora do local de trabalho, em vez de exposições a pacientes com COVID-19, podem ser os principais impulsionadores da infecção por SARS-CoV-2 entre profissionais nos Estados Unidos. Ressaltaram ainda que esses achados sugerem que as medidas atuais de controle de infecção são eficazes para prevenir a transmissão da SARS-CoV-2 ao trabalhar com pacientes, e o risco de infecção do profissional de saúde pode ser impulsionado por exposições ocupacionais de cuidados comunitários e não de pacientes囚.

No Brasil, as instituições de vigilância do trabalho apresentaram recomendações sobre condições para a realização das atividades de trabalho voltadas para reduzir o risco de infecção e mortes pelo vírus SARS-CoV-2. As recomendações abordavam meios de minimizar o contágio: 1 - Realização somente das atividades essenciais seguindo as condições obedecidas. 2 - Seguir o comando oficial das autoridades sanitárias municipais e estaduais que estabelecem orientações de medidas de restrição à circulação de pessoas. 3 - Seguir as condições dos locais de trabalho, por meio do estabelecimento e orientação de todos os trabalhadores do plano e protocolos de 
medidas preventivas. 4 - E Proteção aos

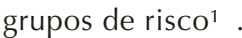

Muitos dos protocolos de coleta de dados se referem ao início da pandemia no Brasil, devido a urgência de levantamento de informações sobre a nova doença, os setores, secretarias, órgãos representativos se apressaram em produzir materiais de orientação e direcionamento para atuação profissional, adaptações nos processos de trabalho. É possível que não represente o cenário atual porque, apesar de relativamente pouco tempo, muitas mudanças aconteceram de forma significativa. A melhor distribuição dos EPIs, maior conhecimento da doença, tratamento, epidemiologia e o avanço da vacina são fatores fundamentais para gerar mudanças na perspectiva que anteriormente assombrava o cenário da saúde do trabalhador, como ocorreu há um ano atrás.

Espera-se que com os dois anos de pandemia a população já esteja mais adaptada com os protocolos, normas, diretrizes técnicas e as medidas de precaução e prevenção da COVID-19. No entanto, é importante considerar o que alguns estudos alertam sobre o risco de contaminação. Pois pode ocorrer no momento em que o profissional está relaxado das atitudes de segurança que são geralmente exigidas no ambiente laboral. Fora desses ambientes, os profissionais ao deixarem de seguir as condutas de segurança se expõem ao risco. Faz-se importante que os órgãos de saúde e vigilância do trabalho abordem as medidas de segurança no ambiente de trabalho, mas lancem campanhas de sensibilização dos profissionais para que se mantenham vigilantes e continuem com medidas de proteção dentro e fora dos ambientes de trabalho.

Bandyopadhyay, Baticulon e Kadhum (2020) ${ }^{11}$ concluíram que o risco de infecções e mortes por COVID-19 entre os profissionais de saúde seguem as da população em geral em todo o mundo. Relacionado ao risco de contágio, afirmam que embora profissionais de certas especialidades possam ser considerados de alto risco devido à exposição às secreções oronasais, por exemplo, o risco para outras especialidades não deve ser subestimado. De forma que se faz necessário refletir sobre as condições de trabalho dos profissionais de enfermagem no enfren- tamento da COVID-19 e repensar as ações e políticas públicas sobre a vigilância e a saúde do trabalhador.

\section{CONCLUSÃO}

Os achados dos estudos reforçam o quanto é importante a Vigilância em Saúde do Trabalhador manter atuação contínua e sistemática, ao longo do tempo, com a finalidade de planejar, executar e avaliar intervenções sobre os aspectos tecnológico, social, organizacional e epidemiológico envolvendo a saúde do trabalhador. Diante do atual cenário epidemiológico e enfrentamento da pandemia do novo Coronavírus, se torna fundamental que o seu conhecimento propicie compreensão do panorama dos profissionais que estão atuando na assistência à Saúde de forma direta ou indiretamente, no Brasil.

Os dados encontrados demonstram a necessidade de ações protetivas que permitam o cuidado integral à saúde dos profissionais de saúde, com a implementação de protocolos que possam reduzir os riscos de contaminação durante a atividade laboral.

\section{Referências}

1 - BRASIL. COVID-19 Painel Coronavírus. 2009. [Acesso em 20/05/2021, 20:00h] https://covid.saude.gov.br/.

2 - BRASIL. Ministério de Estado da Saúde. Portaria n 3.120, de $1^{\circ}$ de julho de 1998. Plataforma Renast Online. Disponível em: http://renastonline.ensp.fiocruz.br/recursos/ portaria-3120-10-julho-1998. [Acesso em: 4 dez. 2018.]

3 - Backes MTS, Higashi GDC, Damiani PR, Mendes JS, Sampaio LS, Soares GL. Condições de trabalho dos profissionais de enfermagem no enfrentamento da pandemia da covid-19. Rev Gaúcha Enferm. 2021;42(esp):e20200339. doi: https://doi. org/10.1590/1983- 1447.2021.20200339

4 - LIMA-COSTA; FERNANDA, M. Tipos de Estudos epidemiológicos: Conceitos básicos e aplicações na área do envelhecimento. Epidemiologia e Serviços de Saúde. 2003

5 - GHOLAMI, M., FAWAD, I., SHADAN, S., COVID-19 and healthcare workers: A systematic review and meta-analysis. International Journal of Infectious Diseases 104 (2021) 335-346. DOI: https://doi.org/10.1016/j.ijid.2021.01.013 1201-9712/@ 2021 The Authors. Published by Elsevier Ltd on behalf of International Society for Infectious Diseases. This is an open access article under the CC BY-NC-ND license (http://creativecommons. org/licenses/by-nc-nd/4.0/)

6 - LIMA, Y. O., COSTA, D. M., SOUZA, J. M. Risco de Contágio por Ocupação no Brasil. Impacto COVID-19, Rio de Janeiro, 26 de Mar. de 2020. Disponível em: https://impactocovid.com.br. Acesso em: 10 de Jul. de 2021

7 - MIRANDA, Fernanda Moura D'Almeida et al. CONDIÇÕES DE TRABALHO E O IMPAC-

TO NA SAÚDE DOS PROFISSIONAIS DE ENFERMAGEM FRENTE A COVID-19. Cogitare

Enfermagem, [S.I.], v. 25, maio 2020. ISSN 2176-9133. Disponível em: <https://revis- tas.ufpr.br/cogitare/article/view/72702>. Acesso em: 04 ago. 2021. doi:http://dx.doi. org/10.5380/ce.v25i0.72702.

8 - PRAXEDES. A., et al. (2021). Análise da morbimortalidade dos casos da COVID-19 nos estados brasileiros. Revista Eletrônica Acervo Saúde, 13(2), e6332. https://doi. org/10.25248/reas.e6332.2021

9 - RAFAEL., R. M. R. et al. Epidemiologia, políticas públicas e pandemia de COVID-19: o que esperar no Brasil? Rev. Enferm.. Rio de Janeiro, UERJ, 2020, v.28, p.49570. DOI: http://dx.doi.org/10.12957/reuerj.2020.49570.

10 - SANTOS, Ubiratan, Maeno, Maria. Cuidados com a saúde dos trabalhadores no contexto da pandemia de COVID-19. Postado em: 3 de dezembro de 2020. Disponível em: http://observatoriodamedicina.ensp.fiocruz.br/cuidados-com-a-saude-dos-trabaIhadores-no-contexto-da-pandemia-de-covid-19-por-ubiratan-de-paula-santos-e-maria-maeno

11 - DIÁRIO OFICIAL DA UNIÃO. Publicado em: 01/09/2020 | Edição: 168 | Seção: 1 | Página: 40 Órgão: Ministério da Saúde/Gabinete do Ministro PORTARIA № 2.309, DE 28 DE AGOSTO DE 2020 HTTPS://WWW.IN.GOV.BR/EN/WEB/DOU/-/PORTARIA-N-2. 309-DE-28-DE-AGOSTO-DE-2020-275240601

11 - Bandyopadhyay S, Baticulon RE, Kadhum M, et al. Infection and mortality of healthcare workers worldwide from COVID-19: a systematic review. BMJ Glob Health 2020;5(12):e003097. doi:10.1136/bmjgh-2020-003097

12 - Organização Pan-Amaricana da Saúde-OPAS [homepage na Internet]. Histórico da pandemia de COVID-19. [acesso em 08 maio 2021]. Disponível em: https://www.paho. org/pt/covid19/historico-da-pandemia-covid-19 